Pacific Journal of Mathematic 


\title{
THE NILPOTENT PART OF A SPECTRAL OPERATOR
}

\author{
Charles A. McCarthy
}

1. Introduction. Throughout this paper, $\mathfrak{X}$ is a Banach space, $T$ a bounded spectral operator on $\mathfrak{X}$ with scalar part $S$, nilpotent part $N$, and resolution of the identity $E(\sigma)$ for $\sigma$ a Borel set in the complex plane. $M$ is the bound for the norms of the $E(\sigma) ;|E(\sigma)| \leq M$ for all Borel sets $\sigma$. The resolvent function for $T,(\lambda-T)^{-1}$, is denoted by $R(\lambda, T)$. The operator $R(\lambda, T) E(\sigma)$ has an unique analytic extension from the resolvent set of $T$ to the complement of $\bar{\sigma}$, and on the subspace $E(\sigma) \mathfrak{X}$ it is equal to the operator $R\left(\lambda, T_{\sigma}\right)$ where $T_{\sigma}$ is the restriction of $T$ to $E(\sigma) \mathfrak{X}$. For material on spectral operators, we refer to the papers on N. Dunford [1], [2]. $\chi_{\sigma}(\xi)$ is the characteristic function of the Borel set $\sigma: \chi_{\sigma}(\xi)=1$ if $\xi \in \sigma, \chi_{\sigma}(\xi)=0$ if $\xi \notin \sigma$. For $p$ a nonnegative real number, $\mu_{p}$ is Hausdorff $p$-dimensional measure [3, pp. 102 ff.]; $\mu_{2}$ is Lebesgue planar measure multiplied by $\pi / 4$, and $\mu_{1}$ restricted to an arc is majorized by arc length.

We assume throughout that there is an integer $m$ for which the resolvent function for $T$ satisfies the $m$ th order rate of growth condition

$$
|R(\lambda, T) E(\sigma)| \leq K \cdot d(\lambda, \sigma)^{-m}, \lambda \notin \bar{\sigma},|\lambda| \leq|T|+{ }^{*} 1,
$$

where $d(\lambda, \sigma)$ is the distance from $\lambda$ to $\sigma$ and $K$ is a constant independent of $\sigma$. If $\mathfrak{X}$ is Hilbert space, it is known that this growth condition implies $N^{m}=0$ [1, p. 337]. In an arbitrary Banach space, this is no longer true; the best that can be done is $N^{m+2}=0$. If $\mathfrak{X}$ is weakly complete, $N^{m+1}=0$; or if $\sigma$ is a set of $\mu_{2}$ measure zero, $N^{m+1} E(\sigma)=0$. If $\sigma$ lies in an arc and either $\mathfrak{X}$ is weakly complete or $\sigma$ has $\mu_{1}$ measure zero, then $N^{m} E(\sigma)=0$. Examples show that we cannot obtain lower indices of nilpotency in general.

2. The fundamental lemma and some easy consequences. If $f(\xi)$ is a bounded, scalar valued Borel function, the operator $\int f(\xi) E(d \xi)$ exists as a bounded operator with norm at most $4 M \cdot \sup _{\xi}|f(\xi)|[1, \mathrm{p} .341]$, so that uniform convergence of a sequence of bounded Borel functions $f_{n}(\xi)$ implies convergence in the uniform operator topology of the operators $\int f_{n}(\xi) E(d \xi)$. Thus for a given bounded Borel function $f(\xi)$ and a given positive number $\eta$, there exist a finite number of disjoint Borel sets $\sigma_{i}$ and points $\xi_{i} \in \sigma_{i}$ such that

Received February 5, 1959. This paper is a portion of a doctoral dissertation presented to Yale University, written under the direction of Professor E. Hille, while the author was an NSF fellow. Particular thanks are due to W. G. Bade who read the manuscript and discovered an error in the author's original proof of Theorem 3.1. 


$$
\left|\int f(\xi) E(d \xi)-\sum_{i} f\left(\xi_{i}\right) E\left(\sigma_{i}\right)\right|<\eta
$$

Similiarly if $A_{n}$ are a finite number of bounded operators and $f_{n}(\xi)$ are bounded Borel functions, for any positive number $\eta$, there exist a finite number of disjoint Borel sets $\sigma_{i}$ and points $\xi_{i} \in \sigma_{i}$ such that

$$
\left|\sum_{n} \int A_{n} f_{n}(\xi) E(d \xi)-\sum_{i} \sum_{n} A_{n} f_{n}\left(\xi_{i}\right) E\left(\sigma_{i}\right)\right|<\eta ;
$$

in particular, for an integer $k$ and a positive number $\eta$, there exist a finite number of disjoint Borel sets $\sigma_{i}$ and points $\xi_{i} \in \sigma_{i}$ such that

$$
\left|\int(T-\xi)^{k} E(d \xi)-\sum_{i}\left(T-\xi_{i}\right)^{k} E\left(\sigma_{i}\right)\right|<\eta \text {. }
$$

Lemma 2.1. There exist constants $M_{k}$ such that $\left|N^{k} E(\sigma)\right| \leq M_{k} \in^{k+1-m}$ for any choice of $\varepsilon, 0<\varepsilon \leq 1$, and Borel set $\sigma$ of diameter no greater than $\varepsilon$.

Proof. Pick $\varepsilon, 0<\varepsilon \leq 1$, and let $\sigma$ be any Borel set of diameter no greater than $\varepsilon$. We have [1, p, 338]

$$
N^{k} E(\sigma)=\int_{\sigma}(T-\xi)^{k} E(d \xi)
$$

For any positive number $\eta$, there is a decomposition of $\sigma$ into a finite number of disjoint Borel sets $\sigma_{i} \subset \sigma$, and points $\xi_{i} \in \sigma_{i}$ such that

$$
\left|\int(T-\xi)^{k} E(d \xi)-\sum_{i}\left(T-\xi_{i}\right)^{k} E\left(\sigma_{i}\right)\right|<\eta .
$$

Since $\sigma$ is of diameter at most $\varepsilon$, there is a circle $\Gamma$ of diameter $3 \varepsilon$ which encloses $\sigma$ and for which $|\gamma-\xi| \geq \varepsilon$ for all $\gamma \in \Gamma$ and $\xi \in \sigma$. Then

$$
\left(T-\xi_{i}\right)^{k} E\left(\sigma_{i}\right)=\frac{1}{2 \pi i} \int_{\Gamma}(\gamma-\xi)^{k} R(\gamma, T) E\left(\sigma_{i}\right) d \gamma,
$$

so that

$$
\sum_{i}\left(T-\xi_{i}\right)^{k} E\left(\sigma_{i}\right)=\frac{1}{2 \pi i} \int_{F} R(\gamma, T) \sum_{i}\left(\gamma-\xi_{i}\right)^{k} E\left(\sigma_{i}\right) d \gamma,
$$

which in norm in no greater than

$$
\frac{1}{2 \pi} \cdot \sup _{\gamma \in F}|R(\gamma, T) E(\sigma)| \cdot \sup _{\gamma \in F}\left|\sum_{i}\left(\gamma-\xi_{i}\right)^{k} E\left(\sigma_{i}\right)\right| \cdot \text { length of } \Gamma \text {. }
$$

The $m$ th order rate of growth condition gives 


$$
\sup _{\gamma \in F}|R(\gamma, T) E(\sigma)| \leq K \varepsilon^{-m}
$$

For any $\gamma \in \Gamma$,

$$
\left|\sum_{i}\left(\gamma-\xi_{i}\right)^{k} E\left(\sigma_{i}\right)\right| \leq 4 M \cdot \max _{i}\left|\gamma-\xi_{i}\right|^{k} \leq 4 M(2 \varepsilon)^{k},
$$

so that $(*)$ is no greater than

$$
\frac{1}{2 \pi} K \varepsilon^{-m} \cdot 4 M(2 \varepsilon)^{k} \cdot 6 \pi \varepsilon=M_{k} \varepsilon^{k+1-m},
$$

where $M_{k}=3 \cdot 2^{k+2} K M$, and is independent of $\eta, \varepsilon, \sigma$, and the manner in which $\sigma$ is decomposed. Thus

$$
\left|N^{k} E(\sigma)\right| \leq M_{k} \varepsilon^{k+1-m}+\eta
$$

for every positive $\eta$, which proves the lemma.

THEOREM 2.2. Let $\sigma$ be a Borel set whose Hausdorff p-measure is zero for a given $p$. Then $N^{k} E(\sigma)=0$ where $k$ is an integer and $k \geq p+m-1$.

Proof. Since $\sigma$ has $p$-measure zero, for every $\varepsilon>0$, there is a covering of $\sigma$ by disjoint sets $\sigma_{i}$ of diameter $\varepsilon_{i}$ such that $\sum_{i} \varepsilon_{i}^{p}<\varepsilon$. By Lemma 2.1 we have

$$
\begin{aligned}
\left|N^{k} E(\sigma)\right| & \leq \sum_{i}\left|N^{k} E\left(\sigma_{i}\right)\right| \leq M_{k} \sum_{i} \varepsilon_{i}^{k+1-m} \\
& \leq M_{k} \sum_{i} \varepsilon_{i}^{(p+m-1)+1-m} \leq M_{k} \sum_{i} \varepsilon_{i}^{p} \leq M_{k} \varepsilon .
\end{aligned}
$$

Since $\varepsilon$ may be chosen arbitrarily small, $N^{k} E(\sigma)=0$.

COROLlaRy 2.3. $\quad N^{m+2}=0$.

Proof. Taking $\sigma$ to be the spectrum of $T$ and $p=3, N^{m+2} E(\sigma(T))=0$; but $E(\sigma(T))$ is the identity mapping on $\mathfrak{X}$.

CoRollaRY 2.4. If $\sigma$ has planar measure zero, then $N^{m+1} E(\sigma)=0$.

CoRollary 2.5. If $\sigma$ has $\mu_{1}$-measure zero, then $N^{m} E(\sigma)=0$.

3. The case of weakly complete $\mathfrak{X}$. Let $\sigma$ be a Borel set in the plane. For any $\varepsilon>0$, we can cover $\sigma$ with disjoint Borel sets. $\sigma_{i}$ of diameter $\varepsilon_{i}, \varepsilon_{i} \leq 1$, such that

$$
\sum_{i} \varepsilon_{i}^{2} \leq \mu_{2}(\sigma)+\varepsilon .
$$

Thus by Lemma 2.1, 


$$
\begin{aligned}
\left|N^{m+1} E(\sigma)\right| & \leq \sum_{i}\left|N^{m+1} E\left(\sigma_{i}\right)\right| \leq M_{m+1} \sum_{i} \varepsilon_{i}^{2} \\
& \leq M_{m+1}\left(\mu_{2}(\sigma)+\varepsilon\right) .
\end{aligned}
$$

Since $\varepsilon$ and $\sigma$ are arbitrary, we have for all Borel sets $\sigma$,

$$
\left|N^{m+1} E(\sigma)\right| \leq M_{m+1} \mu_{2}(\sigma) .
$$

As a consequence, all the scalar measures $x^{*} N^{m+1} E(\cdot) x=\left[\left(N^{*}\right)^{m+1} E^{*}(\cdot) x^{*}\right] x$, $x \in \mathfrak{X}, x^{*} \in \mathfrak{X}^{*}$, are absolutely continuous with respect to $\mu_{2}$, and have derivative bounded by $M_{m+1}\left|x^{*}\right||x|$.

Suppose that $f(\xi)=\sum_{p=1}^{P} \alpha_{p} \chi_{\sigma_{p}}(\xi)$ is a simple Borel function; $\alpha_{p}$ are scalar constants and $\sigma_{p}$ are disjoint Borel sets. We have

$$
\begin{aligned}
\left|\int f(\xi)\left(N^{*}\right)^{m+1} E^{*}(d \xi)\right| & \leq \sum_{p=1}^{P}\left|\alpha_{p}\left(N^{*}\right)^{m+1} E^{*}\left(\sigma_{p}\right)\right| \\
& \leq \sum_{p=1}^{P}\left|\alpha_{p}\right| M_{m+1} \mu_{2}\left(\sigma_{p}\right) \\
& =M_{m+1}|f|_{L_{1}\left(\mu_{2}\right)} .
\end{aligned}
$$

Thus if $f_{n}(\xi)$ are simple Borel functions converging in $L_{1}\left(\mu_{2}\right)$ to $f(\xi)$, the operators $\int_{n}^{n} f_{n}(\xi)\left(N^{*}\right)^{m+1} E^{*}(d \xi)$ converge in the uniform operator topology to an operator which we denote by $\int f(\xi)\left(N^{*}\right)^{m+1} E^{*}(d \xi)$; this limit operator has norm bounded by $M_{m+1}|f| \dot{L}_{1}\left(\mu_{2}\right)$.

THEOREM 3.1. If $\mathfrak{X}$ is weakly complete, then $N^{m+1}=0$.

Proof. Assume that $N^{m+1} \neq 0$, so that also $\left(N^{*}\right)^{m+1} \neq 0$. We will first obtain a bicontinuous map of an infinite dimensional $L_{1}$ space into $\mathfrak{X}^{*}$. An analogous map into $\mathfrak{X}$ would show then that $\mathfrak{X}$ cannot be reflexive, since the image in $\mathfrak{X}$ of this $L_{1}$ space would be a closed nonreflexive subspace of $\mathfrak{X}$; however, the map into $\mathfrak{X}^{*}$ is needed for the slightly more general case of $\mathfrak{X}$ weakly complete.

Let the Borel set $\sigma, x_{0} \in \mathfrak{X}$, and $x_{0}^{*} \in \mathfrak{X}^{*}$ be chosen so that $\left[\left(N^{*}\right)^{m+1} E^{*}(\sigma) x_{0}^{*}\right] x_{0} \neq 0$, and let the derivative of the measure $\left[\left(N^{*}\right)^{m+1} E^{*}(\cdot) x_{0}^{*}\right] x_{0}$ be denoted by $g(\xi)$. We can then find a subset $\tau$ of $\sigma$ and a constant $a>0$ such that $\mu_{2}(\tau)>0$ and $|g(\xi)| \geq a$ on $\tau$.

Define the map $\Phi$ of $L_{1}\left(\tau, \mu_{2}\right)$ into $\mathfrak{X}^{*}$ by

$$
\Phi(f)=\int_{\tau} f(\xi)\left(N^{*}\right)^{m+1} E^{*}(d \xi) x_{0}^{*} .
$$

$\Phi$ is a linear map with bound $M_{m+1}\left|x_{0}^{*}\right|$. Now take

$$
x=\int_{\tau}[g(\xi)]^{-1} \operatorname{sgn} \overline{f(\xi)} E(d \xi) x_{0} ;
$$


The norm of $x$ is no greater than $4 M \cdot a^{-1} \cdot\left|x_{0}\right|$. But we have

$$
\begin{aligned}
{[\Phi(f)](x) } & =\int_{\tau} f(\xi)[g(\xi)]^{-1} \operatorname{sgn} \overline{f(\xi)}\left[\left(N^{*}\right)^{m+1} E^{*}(d \xi) x_{0}^{*}\right] x_{0} \\
& =\int_{\tau}|f(\xi)|[g(\xi)]^{-1} g(\xi) \mu_{2}(d \xi) \\
& =|f|_{L_{1}},
\end{aligned}
$$

which shows that

$$
|\Phi(f)| \geq|f|_{L_{1}} \cdot a \cdot\left(4 M\left|x_{0}\right|\right)^{-1},
$$

so that $\Phi$ is one-to-one and has a continuous inverse.

Now let $\Psi$ be the map of $L_{\infty}\left(\tau, \mu_{2}\right)$ into $\mathfrak{X}$ :

$$
\Psi(h)=\int_{\tau}[g(\xi)]^{-1} h(\xi) E(d \xi) x_{0},
$$

$\Psi$ is a continuous map with bound no greater than $4 M \cdot a^{-1}\left|x_{0}\right|$; we will show that $\Psi$ is one-to-one and bicontinuous. We have

$$
\begin{aligned}
\Phi(f) \Psi(h) & =\int_{\tau} f(\xi)[g(\xi)]^{-1} h(\xi)\left[\left(N^{*}\right)^{m+1} E^{*}(d \xi) x_{0}^{*}\right] x_{0} \\
& =\int_{\tau} f(\xi) h(\xi) \mu_{2}(d \xi)
\end{aligned}
$$

so that

$$
\begin{aligned}
\sup _{|f|_{L_{1}} \leq 1}|\Phi(f) \Psi(h)| & =\sup _{|f|_{L_{1}} \leq 1}\left|\int_{\tau} f(\xi) h(\xi) \mu_{2}(d \xi)\right| \\
& =|h|_{L_{\infty}} .
\end{aligned}
$$

But since $\Phi$ is bounded,

$$
\begin{aligned}
& \sup _{|f|_{L_{1}} \leq 1}|\Phi(f) \Psi(h)| \leq \sup _{\substack{x^{*} \in X^{*} \\
\left|x^{*}\right| \leq|\Phi|}}\left|x^{*} \Psi(h)\right| \\
&=|\Phi||\Psi(h)|,
\end{aligned}
$$

so that

$$
|h|_{L_{\infty}} \leq|\Phi||\Psi(h)|
$$

thus $\Psi$ is one-to-one and bicontinuous, The range $\mathfrak{Y}$ of $\Psi$ in $\mathfrak{X}$ is then a closed non weakly complete subspace of $\mathfrak{X}$. But this is impossible, because every closed subspace of a weakly complete Banach space is again weakly complete; the proof of this last remark is as follows.

Let $\mathfrak{X}$ be a weakly complete Banach space, $\mathfrak{Y}$ a closed subspace. Let $y_{n}$ be a weakly Cauchy sequence in $\mathfrak{Y}$, so that $y^{*} y_{n}$ is a Cauchy sequence of numbers for every $y^{*}$ in $Y^{*}$. Since any $x^{*}$ in $X^{*}$, when 
restricted to $\mathfrak{Y}$, is an element of $\mathfrak{Y}^{*}, x^{*} y_{n}$ is a Cauchy sequence of numbers for every $x^{*}$ in $\mathfrak{X}^{*}$. Since $\mathfrak{X}$ is weakly complete, there is an $x_{0}$ in $\mathfrak{X}$ such that $\lim _{n \rightarrow \infty} x^{*} y_{n}=x^{*} x_{0}$ for every $x^{*}$ in $\mathfrak{X}^{*}$; and since $\mathfrak{Y}$ is strongly closed in $\mathfrak{X}$, it is weakly closed, so that $x_{0}$ must lie in $\mathfrak{Y}$. Finally since every $y^{*}$ in $\mathfrak{Y}^{*}$ is, by the Hahn-Banach theorem, the restriction of an $x^{*}$ in $\mathfrak{X}^{*}, \lim y^{*} y_{n}=y^{*} x_{0}$ for every $y^{*}$ in $\mathfrak{Y}^{*}$, so that $\mathfrak{Y}$ is weakly complete.

THEOREM 3.2. If $\mathfrak{X}$ is weakly complete, then $N^{m} E(\sigma)=0$ for every set $\sigma$ of finite $\mu_{1}$-measure.

Proof. Follow exactly the same discussion above, replacing the number $m+1$ by $m$ and the measure $\mu_{2}$ by $\mu_{1}$.

Note that Theorems 3.1 and 3.2 also hold if $\mathfrak{X}$ is assume to be separable instead of weakly complete, for the image of the $L_{\infty}$ space in $\mathfrak{X}$ would be a nonseparable closed subspace of $\mathfrak{X}$; but every closed subspace of a separable space is again separable.

4. Examples. In the following examples we will need two computational lemmas.

Lemma 4.1. For each real number $p \geq 1$ and Borel set $\sigma$,

$$
\int_{\tau}|\lambda-\xi|^{-(p+2)} \mu_{2}(d \xi) \leq 8 d(\lambda, \sigma)^{-p} \text {, for all } \lambda \notin \bar{\sigma} \text {. }
$$

Proof.

$$
\begin{aligned}
& \int_{\sigma}|\lambda-\xi|^{-(p+2)} \mu_{2}(d \xi) \\
& \quad \leq \int_{|\lambda-\xi| \geq a(\lambda, \sigma)}|\lambda-\xi|^{-(p+2)} \mu_{2}(d \xi) \\
& \quad=\frac{4}{\pi} \int_{0}^{2 \pi} d \theta \int_{a(\lambda, \sigma)}^{\infty} r^{-(p+2)} r d r \\
& \quad \leq 8 d(\lambda, \sigma)^{-p}
\end{aligned}
$$

Lemma 4.2. For each real number $p \geq 1$ and Borel subset $\sigma$ of the real line,

$$
\int_{\sigma}|\lambda-\xi|^{-(p+1)} \mu_{1}(d \xi) \leq 2^{p+1} \pi d(\lambda, \sigma)^{-p},
$$

where $\mu_{1}$ is Lebesgue measure along the line, and $\lambda$ is any complex number, $\lambda \notin \bar{\sigma}$.

Proof. Let $\lambda=\alpha+i \beta, \alpha, \beta$ real. Then either, (i), $d(\alpha, \sigma) \geq d(\lambda, \sigma) / 2$ or, (ii) $|\beta| \geq d(\lambda, \sigma) / 2$. In case (i) we have 


$$
\begin{aligned}
\int_{\sigma}|\lambda-\xi|^{-(p+1)} \mu_{1}(d \xi) & \leq \int_{a(\lambda, \sigma) / 2}^{\infty} \eta^{-(p+1)} d \eta \quad(\lambda-\xi=\eta) \\
& \leq 2^{p+1} p^{-1} d(\lambda, \sigma)^{-p} .
\end{aligned}
$$

In case (ii) we have

$$
\begin{aligned}
\int_{\sigma}|\lambda-\xi|^{-(p+1)} \mu_{1}(d \xi) & \leq \int_{-\infty}^{\infty}|\xi-i \beta|^{-(p+1)} d \xi \\
& \leq \int_{-\infty}^{\infty}\left(\xi^{2}+\beta^{2}\right)^{-\frac{1}{2}(p+1)} d \xi \\
& \leq 2^{p+1} \pi d(\lambda, \sigma)^{-p} .
\end{aligned}
$$

EXAMPLE 4.3. Let $\Sigma$ be a disc in the plane with $\mu_{2}$-measure 1 . Let

$$
x=L_{\infty}(\Sigma) \oplus L_{2}(\Sigma) \oplus \cdots \oplus L_{2}(\Sigma) \oplus L_{1}(\Sigma),
$$

where $m$ copies of $L_{2}(\Sigma)$ are taken. Let $T$ be the operator $S+N$ where $S$ and $N$ are defined as

$$
\begin{aligned}
& S\left[f(\xi) \oplus g_{1}(\xi) \oplus \cdots \oplus g_{m}(\xi) \oplus h(\xi)\right] \\
& \quad=\left[\xi f(\xi) \oplus \xi g_{1}(\xi) \oplus \cdots \oplus \xi g_{m}(\xi) \oplus \xi h(\xi)\right], \\
& N\left[f(\xi) \oplus g_{1}(\xi) \oplus \cdots \oplus g_{m}(\xi) \oplus h(\xi)\right] \\
& \quad=\left[0 \oplus f(\xi) \oplus g_{1}(\xi) \oplus \cdots \oplus g_{m}(\xi)\right] .
\end{aligned}
$$

Since $\Sigma$ has measure 1, any function in $L_{r}$ is in $L_{s}$ for all $s \leq r$, and the $L_{s}$ norm is no greater than the $L_{r}$ norm; thus $N$ is a bounded operator with norm 1 . Also $N$ is a nilpotent for which $N^{m+1} \neq 0$. The operator $T$ is a spectral operator with resolution of the identity

$$
\begin{aligned}
& E(\sigma)\left[f(\xi) \oplus g_{1}(\xi) \oplus \cdots \oplus g_{m}(\xi) \oplus h(\xi)\right] \\
& \quad=\left[f(\xi) \chi_{\sigma}(\xi) \oplus g_{1}(\xi) \chi_{\sigma}(\xi) \oplus \cdots \oplus g_{m}(\xi) \chi_{\sigma}(\xi) \oplus h(\xi) \chi_{\sigma}(\xi)\right] .
\end{aligned}
$$

The resolvent function is

$$
\begin{aligned}
& R(\lambda, T) E(\sigma)\left[f(\xi) \oplus g_{1}(\xi) \oplus \cdots \oplus g_{m}(\xi) \oplus h(\xi)\right] \\
&=\left[\frac{f(\xi) \chi_{\sigma}(\xi)}{\lambda-\xi} \oplus\left(\frac{g_{1}(\xi) \chi_{\sigma}(\xi)}{\lambda-\xi}+\frac{f(\xi) \chi_{\sigma}(\xi)}{(\lambda-\xi)^{2}}\right) \oplus \cdots \oplus\right. \\
&\left(\frac{g_{m}(\xi) \chi_{\sigma}(\xi)}{\lambda-\xi}+\cdots+\frac{g_{1}(\xi) \chi_{\sigma}(\xi)}{(\lambda-\xi)^{m}}+\frac{f(\xi) \chi_{\sigma}(\xi)}{(\lambda-\xi)^{m+1}}\right) \\
&\left.\oplus\left(\frac{h(\xi) \chi_{\sigma}(\xi)}{\lambda-\xi}+\frac{g_{m}(\xi) \chi_{\sigma}(\xi)}{(\lambda-\xi)}+\cdots+\frac{g_{1}(\xi) \chi_{\sigma}(\xi)}{(\lambda-\xi)^{m+1}}+\frac{f(\xi) \chi_{\sigma}(\xi)}{(\lambda-\xi)^{m+2}}\right)\right] .
\end{aligned}
$$

All the terms are clearly of $m$ th order rate of growth except possibly for 

(a) $\left|\frac{f(\xi) \chi_{\sigma}(\xi)}{(\lambda-\xi)^{m+1}}\right|_{L_{2}}$,
(b) $\left|\frac{f(\xi) \chi_{\sigma}(\xi)}{(\lambda-\xi)^{m+2}}\right|_{L_{1}}$, and
(c) $\left|\frac{g_{1}(\xi) \chi_{\sigma}(\xi)}{(\lambda-\xi)^{m+1}}\right|_{L_{1}}$

For (a) we have

$$
\begin{aligned}
\left\{\int_{\sigma}\left|f(\xi)(\lambda-\xi)^{-(m+1)}\right|{ }^{2} \mu_{2}(d \xi)\right\}^{1 / 2} & \leq|f|_{L_{\infty}}\left\{\int_{\sigma} \lambda-\left.\xi\right|^{-2 m-2} \mu_{2}(d \xi)\right\}^{1 / 2} \\
& \leq|f|_{L_{\infty}} \sqrt{8} d(\lambda, \sigma)^{-m}
\end{aligned}
$$

for (b) we have

$$
\begin{aligned}
\int_{\sigma}\left|f(\xi)(\lambda-\xi)^{-(m+2)}\right| \mu_{2}(d \xi) & \leq|f|_{L_{\infty}} \int_{\sigma}|\lambda-\xi|^{-(m+2)} \mu_{2}(d \xi) \\
& \leq|f|_{L_{\infty}} \cdot 8 d(\lambda, \sigma)^{-m}
\end{aligned}
$$

and for (c) we have

$$
\begin{aligned}
\int_{\sigma}\left|g_{1}(\xi)(\lambda-\xi)^{-(m+1)}\right| \mu_{2}(d \xi) & \leq\left\{\int_{\sigma}\left|g_{1}(\xi)\right|^{2} \mu_{2}(d \xi)\right\}^{1 / 2}\left\{\int_{\sigma}|\lambda-\xi|^{-2 m-2} \mu_{2}(d \xi)\right\}^{1 / 2} \\
& \leq\left|g_{1}\right|_{L_{2}} \cdot \sqrt{8} \cdot d(\lambda, \sigma)^{-m}
\end{aligned}
$$

Thus each term of the resolvent, and hence the resolvent itself satisfies the $m$ th order rate of growth condition; this shows that Corollary 2.3 cannot be improved.

EXAMPLE 4.4. Let $\Sigma$ be as in the previous example and let

$$
\mathfrak{X}=L_{r}(\Sigma) \oplus \cdots \oplus L_{r}(\Sigma) \oplus L_{s}(\Sigma)
$$

where $m$ copies of $L_{r}$ are taken. $r$ and $s$ are to satisfy $1<s<r<\infty$ and $r s \leq 2(r-s)$. Let $T=S+N$, where $S$ and $N$ are defined in essentially the same way as in the previous example. The resolvent function is given by

$$
\begin{aligned}
& R(\lambda, T) E(\sigma)\left[f_{1}(\xi) \oplus \cdots \oplus f_{m}(\xi) \oplus g(\xi)\right] \\
&= {\left[\frac{f_{1}(\xi) \chi_{\sigma}(\xi)}{\lambda-\xi} \oplus \cdots \oplus\left(\frac{f_{m}(\xi) \chi_{\sigma}(\xi)}{\lambda-\xi}+\cdots+\frac{f_{1}(\xi) \chi_{\sigma}(\xi)}{(\lambda-\xi)^{m}}\right)\right.} \\
&\left.\oplus\left(\frac{g(\xi) \chi_{\sigma}(\xi)}{\lambda-\xi}+\frac{f_{m}(\xi) \chi_{\sigma}(\xi)}{(\lambda-\xi)^{2}}+\cdots+\frac{f_{1}(\xi) \chi_{\sigma}(\xi)}{(\lambda-\xi)^{m+1}}\right)\right] .
\end{aligned}
$$

Each of the terms is clearly of $m$ th order rate of growth except possibly for the $L_{s}$ norm of $f_{1}(\xi)(\lambda-\xi)^{-(m+1)} \chi_{\sigma}(\xi)$, and for this we have

$$
\begin{aligned}
& \left\{\int_{\sigma}\left|f_{1}(\xi)(\lambda-\xi)^{m+1}\right|^{s} \mu_{2}(d \xi)\right\}^{1 / s} \\
& \quad \leq\left\{\int_{\sigma}\left|f_{1}(\xi)\right|^{r} \mu_{2}(d \xi)\right\}^{1 / r}\left\{\int|\lambda-\xi|^{-\frac{(m+1) r s}{r-s}} \mu_{2}(d \xi)\right\}^{\frac{r-s}{r s}} \\
& \quad \leq\left|f_{1}\right|_{L_{r}} \cdot 8^{\frac{s-r}{r s}} \cdot d(\lambda, \sigma)^{-m-\left(1-\frac{2(r-s)}{r s}\right)} \\
& \quad \leq\left|f_{1}\right|_{L_{r}} \cdot 8^{\frac{r-s}{r s}} d(\lambda, \sigma)^{-m}
\end{aligned}
$$


Thus the resolvent satisfies the $m$ th order rate of growth condition, and $N^{m}=0$. Since $\mathfrak{X}$ is reflexive, this shows that Theorem 3.1 cannot be improved. Note that $\mathfrak{X}$ is also separable.

EXAMPLE 4.5. Let $\Sigma$ be the interval $[0,1]$ endowed with $\mu_{1}$-measure, and let

$$
\mathfrak{X}=L_{\infty}(\Sigma) \oplus \cdots \oplus L_{\infty}(\Sigma) \oplus L_{1}(\Sigma)
$$

where $m$ copies of $L_{\infty}$ are taken. Let $T=S+N$ where $S$ and $N$ are defined in essentially the same way as in the previous examples. The resolvent function is given by

$$
\begin{aligned}
R(\lambda, & T) E(\sigma)\left[f_{1}(\xi) \oplus \cdots \oplus f_{m}(\xi) \oplus g(\xi)\right] \\
= & {\left[\frac{f_{1}(\xi) \chi_{\sigma}(\xi)}{\lambda-\xi} \oplus \cdots \oplus\left(\frac{f_{m}(\xi) \chi_{\sigma}(\xi)}{\lambda-\xi}+\cdots+\frac{f_{1}(\xi) \chi_{\sigma}(\xi)}{(\lambda-\xi)^{m}}\right)\right.} \\
& \left.\oplus\left(\frac{g(\xi) \chi_{\sigma}(\xi)}{\lambda-\xi}+\frac{f_{m}(\xi) \chi_{\sigma}(\xi)}{(\lambda-\xi)^{2}}+\cdots+\frac{f_{1}(\xi) \chi_{\sigma}(\xi)}{(\lambda-\xi)^{m+1}}\right)\right] .
\end{aligned}
$$

Each of the terms is clearly of $m$ th order rate of growth except for the $L_{1}$ norm of $f_{1}(\xi)(\lambda-\xi)^{-(m+1)} \chi_{\sigma}(\xi)$, and for this we have

$$
\begin{aligned}
\int_{\sigma}\left|f(\xi)(\lambda-\xi)^{-(m+1)}\right| \mu_{1}(d \xi) & \leq|f|_{L_{\infty}} \int_{\sigma}|\lambda-\xi|^{-(m+1)} \mu_{1}(d \xi) \\
& \leq|f|_{L_{\infty}} 2^{m+1} \pi d(\lambda, \sigma)^{-m}
\end{aligned}
$$

Thus we have an example of an operator with spectrum in a rectifiable arc which satisfies the $m$ th order rate of growth condition, but for which $N^{m} \neq 0$.

\section{BIBLIOGRAPHY}

1. N. Dunford, Spectral operators, Pacific J. Math. 4 (1954), 321-354.

2. - A survey of the theory of spectral operators, Bul. AMS 64 (1958), 217-274.

3. Hurewicz and Wallman, Dimension theory, Princeton University press, 1948.

YALE UNIVERSITY

MassachusetTS INSTITUTE OF TEChNOLOGY 



\section{PACIFIC JOURNAL OF MATHEMATICS}

EDITORS

David Gilbarg

Stanford University

Stanford, California

R. A. Beaumont

University of Washington

Seattle 5 , Washington
A. L. Whiteman

University of Southern California

Los Angeles 7, California

L. J. PAIGE

University of California

Los Angeles 24, California

\section{ASSOCIATE EDITORS}

\author{
E. F. BECKENBACH \\ C. E. BURGESS \\ E. HEWITT \\ A. HORN
}

\author{
V. GANAPATHY IYER \\ R. D. JAMES \\ M. S. KNEBELMAN \\ L. NACHBIN
}
I. NIVEN
E. G. STRAUS
T. G. OSTROM
G. SZEKERES
H. L. ROYDEN
F. WOLF
M. M. SCHIFFER
K. YOSIDA

\section{SUPPORTING INSTITUTIONS}

\author{
UNIVERSITY OF BRITISH COLUMBIA \\ CALIFORNIA INSTITUTE OF TECHNOLOGY \\ UNIVERSITY OF CALIFORNIA \\ MONTANA STATE UNIVERSITY \\ UNIVERSITY OF NEVADA \\ OREGON STATE COLLEGE \\ UNIVERSITY OF OREGON \\ OSAKA UNIVERSITY \\ UNIVERSITY OF SOUTHERN CALIFORNIA
}

\author{
STANFORD UNIVERSITY \\ UNIVERSITY OF TOKYO \\ UNIVERSITY OF UTAH \\ WASHINGTON STATE COLLEGE \\ UNIVERSITY OF WASHINGTON \\ $* \quad * \quad *$ \\ AMERICAN MATHEMATICAL SOCIETY \\ CALIFORNIA RESEARCH CORPORATION \\ HUGHES AIRCRAFT COMPANY \\ SPACE TECHNOLOGY LABORATORIES
}

Mathematical papers intended for publication in the Pacific Journal of Mathematics should be typewritten (double spaced), and the author should keep a complete copy. Manuscripts may be sent to any one of the four editors. All other communications to the editors should be addressed to the managing editor, L. J. Paige at the University of California, Los Angeles 24, California.

50 reprints per author of each article are furnished free of charge; additional copies may be obtained at cost in multiples of 50 .

The Pacific Journal of Mathematics is published quarterly, in March, June, September, and December. The price per volume (4 numbers) is $\$ 12.00$; single issues, $\$ 3.50$. Back numbers are available. Special price to individual faculty members of supporting institutions and to individual members of the American Mathematical Society: $\$ 4.00$ per volume; single issues, $\$ 1.25$.

Subscriptions, orders for back numbers, and changes of address should be sent to Pacific Journal of Mathematics, 2120 Oxford Street, Berkeley 4, California.

Printed at Kokusai Bunken Insatsusha (International Academic Printing Co., Ltd.), No. 6, 2-chome, Fujimi-cho, Chiyoda-ku, Tokyo, Japan.

PUBLISHED BY PACIFIC JOURNAL OF MATHEMATICS, A NON-PROFIT CORPORATION

The Supporting Institutions listed above contribute to the cost of publication of this Journal, but they are not owners or publishers and have no responsibility for its content or policies. 


\section{Pacific Journal of Mathematics}

\section{Vol. 9, No. 4 \\ August, 1959}

Frank Herbert Brownell, III, A note on Kato's uniqueness criterion for

Schrödinger operator self-adjoint extensions ............... 953

Edmond Darrell Cashwell and C. J. Everett, The ring of number-theoretic

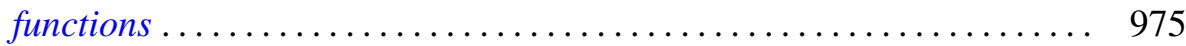

Heinz Otto Cordes, On continuation of boundary values for partial

differential operators ............................. 987

Philip C. Curtis, Jr., n-parameter families and best approximation . . . . . . 1013

Uri Fixman, Problems in spectral operators . . . . . . . . . . . . . . . 1029

I. S. Gál, Uniformizable spaces with a unique structure .............. 1053

John Mitchell Gary, Higher dimensional cyclic elements ............ 1061

Richard P. Gosselin, On Diophantine approximation and trigonometric

polynomials ..................................... 1071

Gilbert Helmberg, Generating sets of elements in compact groups ........ 1083

Daniel R. Hughes and John Griggs Thompson, The H-problem and the

structure of $H$-groups .................................. 1097

James Patrick Jans, Projective injective modules ................. 1103

Samuel Karlin and James L. McGregor, Coincidence properties of birth and

death processes ..................................... 1109

Samuel Karlin and James L. McGregor, Coincidence probabilities ........ 1141

J. L. Kelley, Measures on Boolean algebras ................... 1165

John G. Kemeny, Generalized random variables ................... 1179

Donald G. Malm, Concerning the cohomology ring of a sphere bundle ... . . 1191

Marvin David Marcus and Benjamin Nelson Moyls, Transformations on

tensor product spaces .................................. 1215

Charles Alan McCarthy, The nilpotent part of a spectral operator ........ 1223

Kotaro Oikawa, On a criterion for the weakness of an ideal boundary

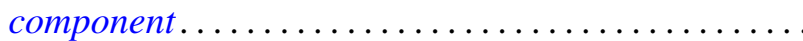

Barrett O'Neill, An algebraic criterion for immersion . . ............... 1239

Murray Harold Protter, Vibration of a nonhomogeneous membrane ... . . . . 1249

Victor Lenard Shapiro, Intrinsic operators in three-space . . . . . . . . . . . 1257

Morgan Ward, Tests for primality based on Sylvester's cyclotomic

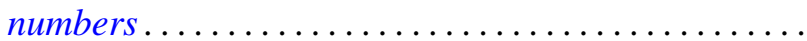

L. E. Ward, A fixed point theorem for chained spaces ....

Alfred B. Willcox, Šilov type $C$ algebras over a connected locally compact

abelian group..................................... 1279

Jacob Feldman, Correction to "Equivalence and perpendicularity of

Gaussian processes" ........................ 